\title{
Efficacy of intraarterial superselective indocyanine green videoangiography in cerebral arteriovenous malformation surgery in a hybrid operating room
}

\author{
Kenji Shimada, MD, PhD, ${ }^{1}$ Tadashi Yamaguchi, MD, ${ }^{1}$ Takeshi Miyamoto, MD, PhD, ${ }^{1}$ \\ Shu Sogabe, MD, PhD, ${ }^{1}$ Masaaki Korai, MD, PhD, ${ }^{2}$ Toshiyuki Okazaki, MD, PhD, ${ }^{3}$ \\ Yasuhisa Kanematsu, MD, PhD, ${ }^{1}$ Junichiro Satomi, MD, PhD, ${ }^{4}$ Shinji Nagahiro, MD, PhD, ${ }^{5}$ and \\ Yasushi Takagi, MD, PhD1
}

\begin{abstract}
${ }^{1}$ Department of Neurosurgery, Tokushima University Hospital, Tokushima; ${ }^{2}$ Department of Neurosurgery, Shikoku Medical Center for Children and Adults, Zentsuji, Kagawa; ${ }^{3}$ Department of Spine, Spinal Cord, Peripheral Nerve Surgery, Shin-yurigaoka General Hospital, Kawasaki, Kanagawa; ${ }^{4}$ Department of Neurosurgery, Kitajima Taoka Hospital; and ${ }^{5}$ Department of Neurosurgery, Yoshinogawa Hospital, Itano, Tokushima, Japan
\end{abstract}

\begin{abstract}
OBJECTIVE Although intravenous indocyanine green (ICG) videoangiography has been reported to be useful when applied to cerebral arteriovenous malformation (AVM) surgery, the ICG that remains after the procedure makes it difficult to understand the anatomy, to evaluate nidus blood flow changes, and to repeat ICG videoangiography within a short time. Intraarterial ICG videoangiography has emerged as a way to overcome these limitations. The current study presents the results of intraarterial ICG videoangiography undertaken in patients with cerebral AVMs.
\end{abstract}

METHODS Intraarterial ICG videoangiography was performed in 13 patients with cerebral AVMs. Routine intraoperative digital subtraction angiography at the authors' institution is performed in a hybrid operating room during AVM surgery and includes the added step of injecting ICG to the contrast medium that is administered through a catheter.

RESULTS Predissection studies were able to visualize the feeder in 12 of 13 cases. The nidus was visualized in 12 of 13 cases, while the drainer was visualized in all cases. After total dissection of the nidus, there was no ICG filling in the drainers found in any of the cases. Washout of the ICG took $4.4 \pm 1.3$ seconds in the feeders, $9.2 \pm 3.5$ seconds in the drainers, and $20.9 \pm 3.4$ seconds in all of the vessels. Nidus flow reduction was confirmed during dissection in 9 of 9 cases. Flow reduction was easy to recognize due to each span being very short. Color-encoded visualization and objective data obtained by Flow 800 analysis reinforced these findings.

CONCLUSIONS The results showed that intraarterial ICG videoangiography was more useful than intravenous ICG videoangiography in cerebral AVM surgery. This was especially effective in the identification of the feeder, nidus, and drainer and in the assessment of the flow dynamics of the nidus. Use of Flow 800 made it simpler and easier to evaluate these findings.

https://thejns.org/doi/abs/10.3171/2020.3.JNS20319

KEYWORDS cerebral arteriovenous malformation; intra-arterial ICG videoangiography; feeder; nidus; drainer; vascular disorders

I NDOCYANINE green (ICG) videoangiography is a frequently used intraoperative technique for vascular neurosurgery due to its integration into operative microscopes. Many previous reports have described the efficacy of ICG videoangiography for cerebral aneurysm, extracranial-intracranial bypass, spinal arteriovenous malformation (AVM)/arteriovenous fistula (AVF), and cere- bral AVM.1-7 However, in almost all reports, these studies used an intravenous injection of ICG. Recently, there have been a few studies that have examined intraarterial injection of ICG, with these reports describing its usefulness for cerebral aneurysm and spinal AVF surgery. ${ }^{8,9}$

Intravenous ICG videoangiography is able to differentiate between the feeder, nidus, and drainer, provided the

ABBREVIATIONS AVF = arteriovenous fistula; $\mathrm{AVM}=$ arteriovenous malformation; $\mathrm{CCA}=$ common carotid artery; $\mathrm{DSA}=$ digital subtraction angiography; $\mathrm{ICG}=$ indocyanine green; $\mathrm{ROI}=$ region of interest; $\mathrm{VA}=$ vertebral artery.

SUBMITTED February 6, 2020. ACCEPTED March 30, 2020.

INCLUDE WHEN CITING Published online May 22, 2020; DOI: 10.3171/2020.3.JNS20319. 
AVM nidus is superficially located and exhibits a reduced nidus flow or residual nidus during or at the end of the resection. ${ }^{2,3,6}$ Although the efficacy of the intravenous injection of ICG for cerebral AVM surgery has been demonstrated, the ICG that remains in the vessels during the late phase makes it difficult to easily understand the anatomical differences that are present and the time-dependent flow dynamic analysis unless the Flow 800 software (Zeiss) is installed in the operative microscope. In addition, because of the large amount of ICG that is required to reach the intracranial arteries due to the dilution in the cardiopulmonary circulation, the long washout time required to remove the ICG means that it is not possible to repeat the ICG videoangiography within a short period of time. However, this type of information is very important during AVM surgeries. Thus, intraarterial injection of ICG has been investigated and shown to be able to overcome the technical limitations of intravenous injections of ICG . ${ }^{8,9}$ Our current study presents the results of intraarterial superselective injections of ICG in a series of cerebral AVM surgeries that was performed in a hybrid operating room. This report also discusses the efficacy of using this particular technique.

\section{Methods \\ Patient Population}

This study was approved by the Tokushima University Institutional Review Board and conducted in compliance with the Health Insurance Portability and Accountability Act regulations. All patients provided written informed consent. ICG videoangiography via intraarterial injection was performed in 13 of 31 patients treated for cerebral AVMs at our institution from 2012 to 2018. Table 1 summarizes the patient characteristics. Indications for ICG videoangiography included AVMs with a nidus and at least 1 drainer located within the superficial surface of the brain.

\section{Intraarterial Injection of ICG Videoangiography}

ICG videoangiography studies were performed using either the OPMI Pentero or Kinevo with integrated ICG technology (Zeiss). Intraoperative digital subtraction angiography (DSA) was routinely performed in our hybrid operating room that was used for the AVM surgeries (Fig. $1 \mathrm{~A}$ and $\mathrm{B}$ ). After general anesthesia, a 5-Fr sheath (Terumo) was inserted in the right femoral artery. A heparincoated 5-Fr catheter was then introduced into the carotid artery or vertebral artery (VA). The catheter was left in the same position until total dissection of the nidus was confirmed. To avoid thromboembolic complications, pressurized $(280 \mathrm{~mm} \mathrm{Hg}) 500-\mathrm{ml}$ bags of heparinized saline $(4 \mathrm{U} / \mathrm{ml})$ were connected to all of the catheters for the purpose of continuous flushing, with manual catheter irrigation performed every 15 minutes (Fig. 1C). The intraoperative DSA study was primarily performed before the dissection and after the total dissection of the AVM. In addition to the intraoperative DSA procedure, $5 \mathrm{ml}$ of ICG (a 2.5-mg dose dissolved in $50 \mathrm{ml}$ of heparinized saline $[4 \mathrm{U} / \mathrm{ml}])$ was injected through the catheter used for the ICG videoangiography. Induction of ICG fluorescence occurred after the dye solution arrived in the vessels of the near-infrared light-illuminated field of interest. Fluorescence (range 780-950 nm; maximum $835 \mathrm{~nm}$ ) was recorded using a nonintensified video camera. An optical filter blocked both the ambient and excitation light so that only the ICG-induced fluorescence was collected. Therefore, this made it possible to observe arterial, capillary, and venous angiographic images in real time on the video screen. Images were recorded on a USB external drive and reviewed later for analysis. Flow 800 software was used to analyze the video obtained during the procedure (Fig. 1D). In addition, the delay map, speed map, and diagram were also recorded and analyzed.

\section{Results}

Of the 13 cases evaluated, ICG videoangiograms detected feeders in 12 cases, a nidus in 12 cases, and drainers in all cases (Table 1). The feeders and nidus that were not detected did exist in the deep and not the superficial parts of the brain. ICG videoangiography was performed in 9 cases after coagulation of the feeders, with a reduced nidus flow confirmed in all cases. ICG videoangiography and DSA were performed in all cases after dissection of the nidus, with no filling of the nidus and drainer confirmed in all of the cases examined (Supplemental Table 1). Washout of ICG took $4.4 \pm 1.3$ seconds $(n=12)$ in feeders, $9.2 \pm 3.5$ seconds in drainers $(\mathrm{n}=13)$, and $20.9 \pm 3.4$ seconds $(n=7)$ in all vessels (Table 2$)$.

\section{Illustrative Cases \\ Case 6}

MRI in a 19-year-old man who presented with head trauma demonstrated an incidental AVM. In addition, DSA showed the presence of a Spetzler-Martin grade II AVM in the right frontal lobe. This was fed by the middle cerebral artery and drained into the ascending cortical veins. The patient underwent a frontotemporal craniotomy, and the nidus was removed after embolization of the feeders and the flow-related aneurysm (Fig. 2A). Intraoperative ICG videoangiography showed that the feeders and nidus appeared at 1 second, after which the feeders disappeared and the drainers appeared at 4 seconds, with the drainers then disappearing at 10 seconds (Fig. 2B-D). After dissection of the superficial part of the nidus (Fig. 2E), ICG videoangiography disclosed that the nidus was still present at 4 seconds, while the drainers were also still present at 10 seconds, which indicated that there was a reduced flow for the nidus (Fig. $2 \mathrm{~F}-\mathrm{H}$ ). After dissection of the nidus (Fig. 2I), ICG videoangiography demonstrated that there was no filling of either the nidus or the drainer (Fig. 2J).

\section{Case 5}

MRI performed in a 42-year-old man who presented with headache demonstrated an incidental AVM. In addition, DSA indicated there was a Spetzler-Martin grade I AVM in the right frontal lobe. This was fed by the anterior cerebral artery, and it drained into the ascending cortical veins. The patient underwent frontal craniotomy, with the 
TABLE 1. Characteristics of patients with AVMs and summary of predissection study of intraarterial ICG videoangiography

\begin{tabular}{|c|c|c|c|c|c|c|}
\hline \multirow[b]{2}{*}{ Case No. } & \multirow[b]{2}{*}{ Age (yrs), Sex } & \multirow[b]{2}{*}{ S-M Grade } & \multirow[b]{2}{*}{ Location (nidus) } & \multicolumn{3}{|c|}{ Visualization (before dissection) } \\
\hline & & & & Feeder & Nidus & Drainer \\
\hline 1 & $15, M$ & III & Occipital & Yes & Yes & Yes \\
\hline 2 & $23, \mathrm{~F}$ & V & Frontal & Yes & Yes & Yes \\
\hline 3 & $43, F$ & II & Parietal & Yes & Yes & Yes \\
\hline 4 & $56, \mathrm{M}$ & II & Parietal & No & Yes & Yes \\
\hline 5 & $42, \mathrm{M}$ & I & Frontal & Yes & Yes & Yes \\
\hline 6 & $19, \mathrm{M}$ & II & Frontal & Yes & Yes & Yes \\
\hline 7 & $31, \mathrm{~F}$ & IV & Parietal & Yes & No & Yes \\
\hline 8 & $45, M$ & 1 & Temporal & Yes & Yes & Yes \\
\hline 9 & $16, M$ & I & Temporal & Yes & Yes & Yes \\
\hline 10 & $38, F$ & I & Cerebellum & Yes & Yes & Yes \\
\hline 11 & $28, M$ & II & Cerebellum & Yes & Yes & Yes \\
\hline 12 & $32, \mathrm{~F}$ & II & Parietal & Yes & Yes & Yes \\
\hline 13 & $32, \mathrm{M}$ & IV & Occipital & Yes & Yes & Yes \\
\hline
\end{tabular}

S-M = Spetzler-Martin.

nidus removed after feeder embolization (Fig. 3A). Intraoperative ICG videoangiography disclosed that the feeders and nidus appeared at 1 second, after which the feeders disappeared and the drainers appeared at 4 seconds, with the drainers then disappearing at 10 seconds (Fig. 3B-D). After clipping the feeder from the frontolateral side (Fig. 3E), ICG videoangiography disclosed filling of the nidus with ICG from the frontomedial side (Fig. 3F). Before removal of the nidus (Fig. 3G), ICG videoangiography showed that the nidus was still filled with ICG (Fig. $3 \mathrm{H}$ ), which demonstrated that the nidus was not completely dissected. The nidus was removed after ICG videoangiography showed that there was no filling of either the nidus or its drainer (Fig. 3I and J).

TABLE 2. Washout time of ICG

\begin{tabular}{cccc}
\hline & \multicolumn{3}{c}{ Washout Time (sec) } \\
\cline { 2 - 4 } Case No. & Feeders & Drainers & All Vessels \\
\hline 1 & 4 & 20 & 25 \\
\hline 2 & 4 & 7 & 15 \\
\hline 3 & 3 & 7 & 18 \\
\hline 4 & $\mathrm{NA}$ & 7 & 22 \\
\hline 5 & 4 & 8 & 22 \\
\hline 6 & 4 & 12 & 19 \\
\hline 7 & 5 & 7 & NA \\
\hline 8 & 4 & 7 & NA \\
\hline 9 & 3 & 7 & NA \\
\hline 10 & 8 & 11 & NA \\
\hline 11 & 5 & 9 & NA \\
\hline 12 & 5 & 10 & 25 \\
\hline 13 & 4 & 8 & NA \\
\hline Mean \pm SD & $4.4 \pm 1.3$ & $9.2 \pm 3.5$ & $20.9 \pm 3.4$ \\
\hline
\end{tabular}

NA = not applicable.

\section{Case 4}

A 56-year-old man presented with generalized seizures. DSA revealed a Spetzler-Martin grade II AVM in the right parietal lobe, which was fed by the anterior cerebral artery and drained into the superior sagittal sinus. The patient underwent parietal craniotomy and the nidus was removed (Fig. 4A). Intraoperative ICG videoangiog-
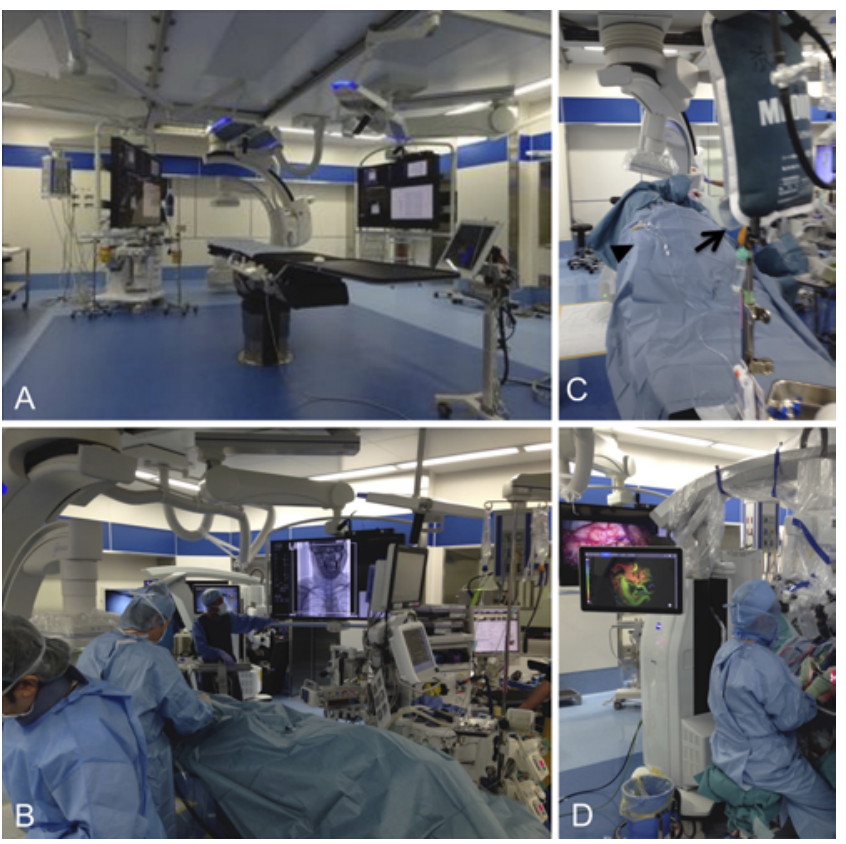

FIG. 1. Photographs of the hybrid operating room (A) and intraoperative DSA unit (B). The pressurized $500-\mathrm{ml}$ bag of heparinized saline for the flush (arrow, C) is connected to the catheter (arrowhead, C) through which the contrast medium or ICG can be injected at any time. Catheter irrigation is performed manually every 15 minutes (C). Flow 800 analysis can provide real-time results (D). 

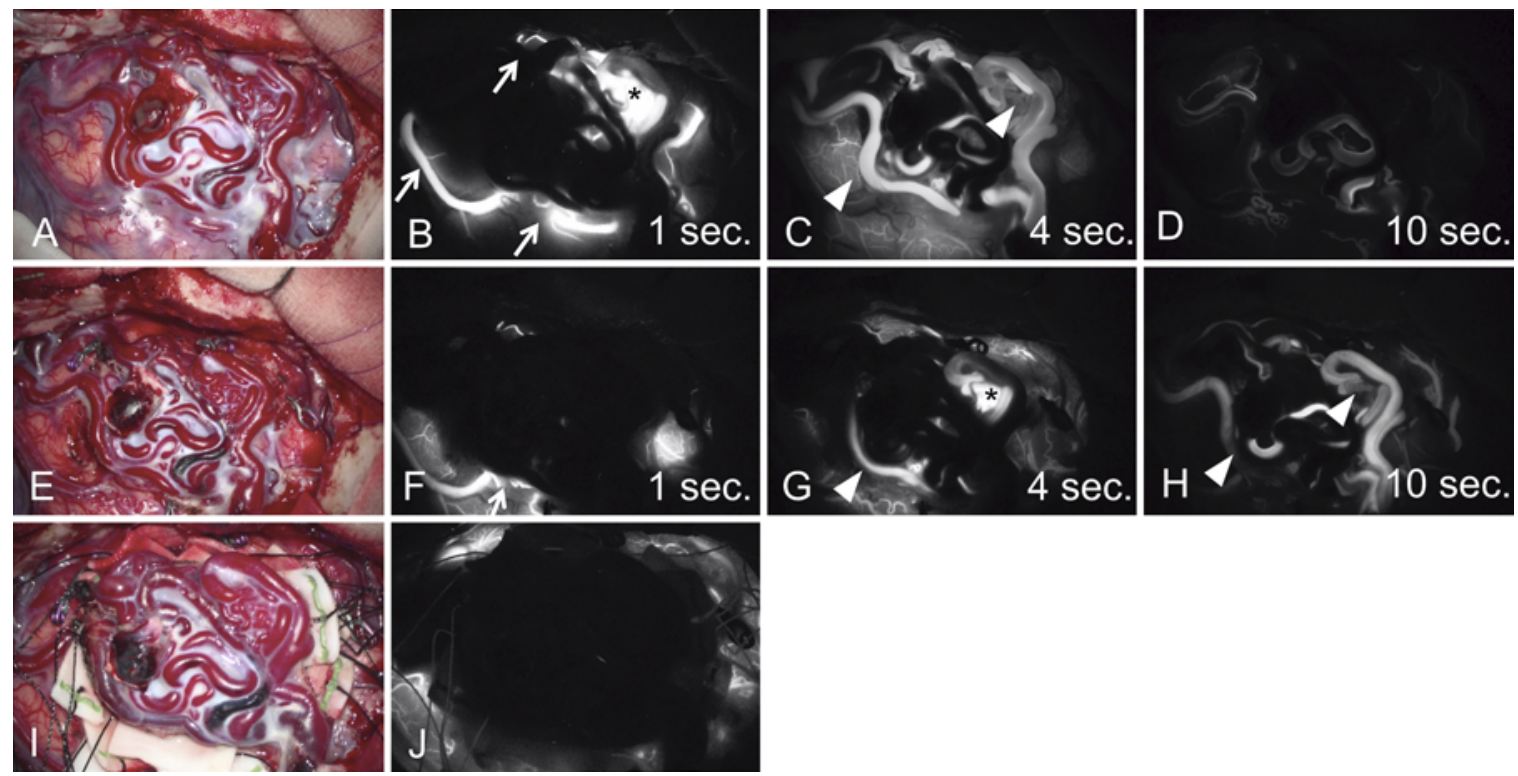

FIG. 2. Case 6. Surgical views before (A) and after partial (E) and total (I) dissection of the nidus, as well as ICG videoangiography studies (B-D, F-H, and J) that were performed at the time of the operation. Intraoperative ICG videoangiography revealed that the feeders and nidus appeared at 1 second, while the feeders disappeared and drainers appeared at 4 seconds, with the drainers then disappearing at 10 seconds (B-D and F-H: arrows indicate the feeder; asterisk indicates the nidus; and arrowheads indicate the drainer). After dissection of the superficial part of the nidus, ICG videoangiography revealed that the nidus still appeared at 4 seconds, while the drainers still appeared at 10 seconds, which indicates that there was a reduced flow of the nidus (F-H). After dissection of the nidus, ICG videoangiography revealed there was no filling of the nidus and drainer $(\mathrm{J})$.
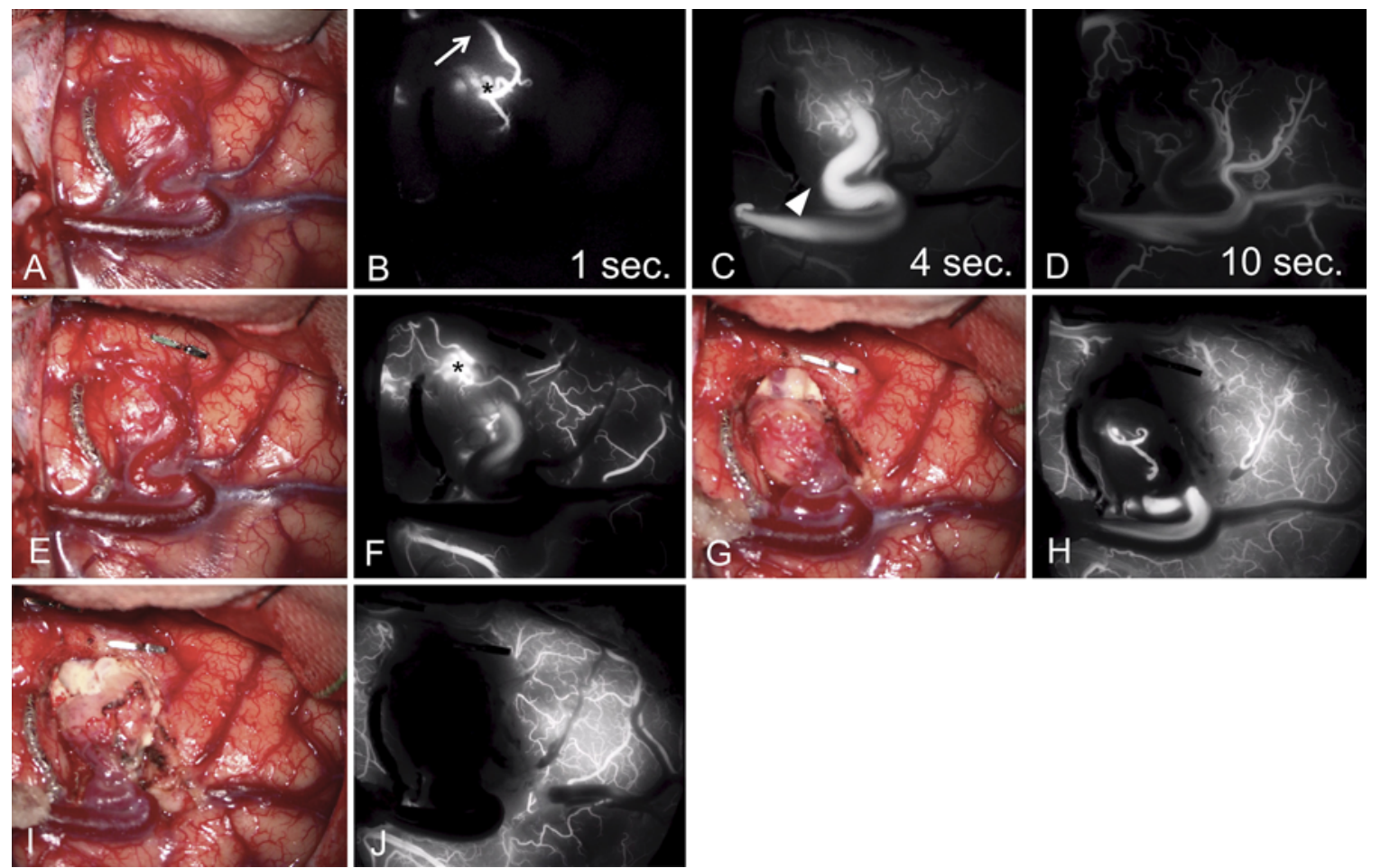

FIG. 3. Case 5. Surgical view before dissection (A), after clipping of the feeder (E), after dissection $(\mathbf{G})$ and after complete dissection (I) of the nidus, as well as ICG videoangiography studies (B-D, F, H, and J) that were performed at the time of the operation. Intraoperative ICG videoangiography disclosed that the feeders and nidus appeared at 1 second, while the feeders disappeared and drainers appeared at 4 seconds, with the drainers then disappearing at 10 seconds (B-D and F: arrow indicates the feeder; asterisk indicates the nidus; and arrowhead indicates the drainer). After clipping of the feeder from the frontolateral side, ICG videoangiography revealed that the nidus was filled with ICG from the frontomedial side (E and F). ICG videoangiography revealed that the nidus was still filled with ICG $(\mathrm{H})$. ICG videoangiography revealed that there was no filling of the nidus and its drainer (J). 

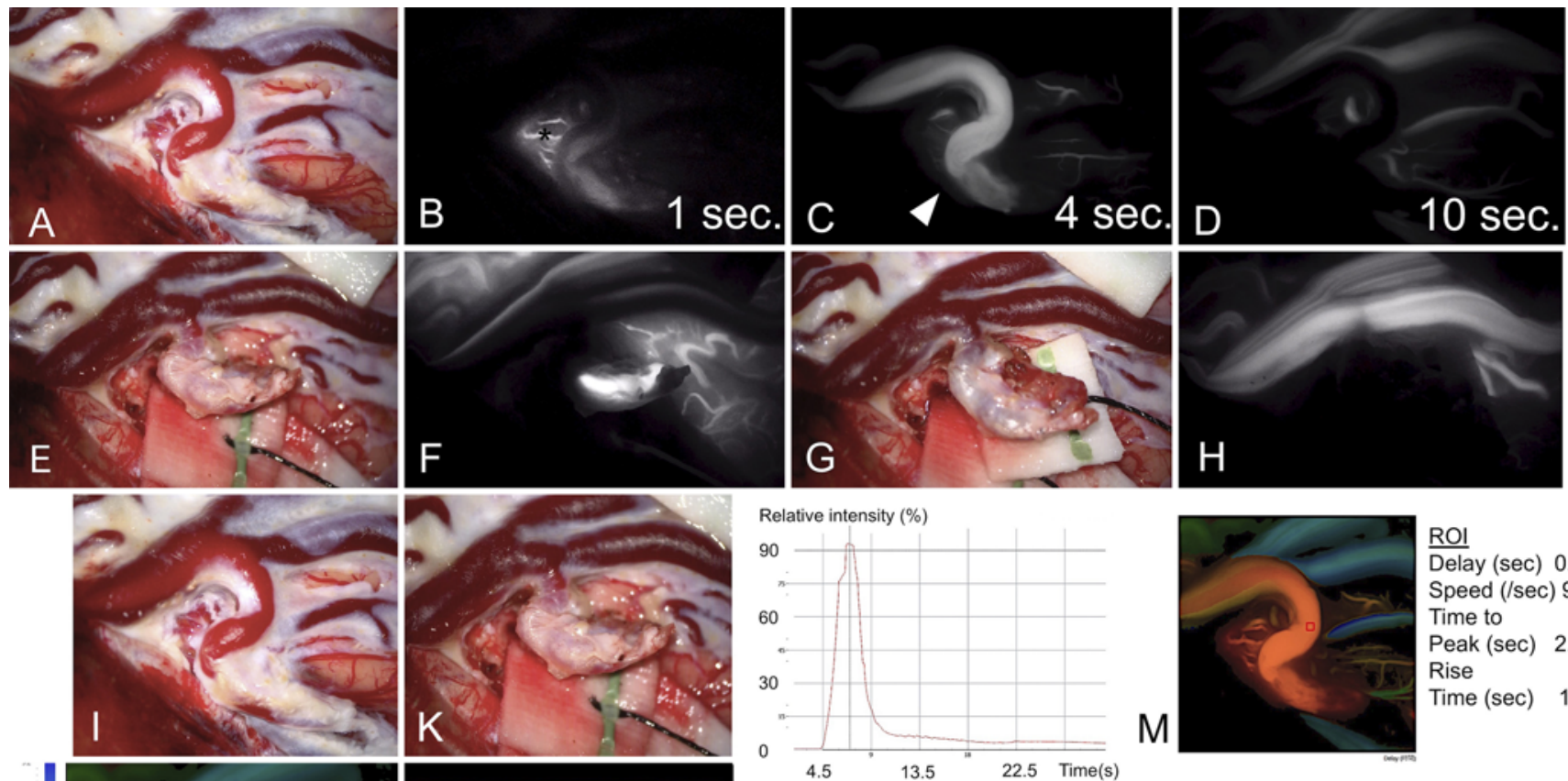

$\frac{\mathrm{ROI}}{\text { Delay (sec) } 0.64}$ Speed (/sec) 944 Time to Peak (sec) 2.61 Rise Time (sec) $\quad 1.82$
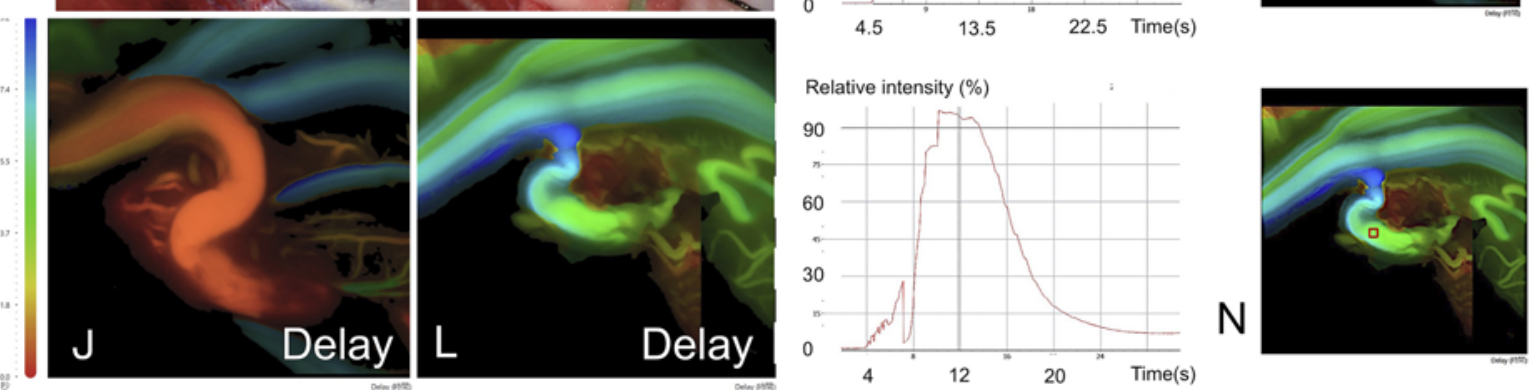

ROI

Delay (sec) 4.74 Speed (/sec) 159 Time to

Peak (sec) 7.89

Rise

Time (sec) $\quad 4.8$

FIG. 4. Case 4. Surgical view before dissection (A), after dissection (E), and after complete dissection (G) of the nidus, as well as ICG videoangiography studies (B-D, F, and H) that were performed at the time of the operation. Intraoperative ICG videoangiography revealed that the nidus appeared at 1 second, while the drainers appeared at 4 seconds and then disappeared at 10 seconds (B-D: asterisk indicates the nidus and arrowhead indicates the drainer). ICG videoangiography revealed that the nidus was still filled with ICG $(F)$. ICG videoangiography revealed that there was no filling of the nidus and its drainer $(H)$. The captured video was analyzed by Flow 800 . Surgical views before $(\mathbf{I})$ and after $(\mathbf{K})$ dissection of the nidus. The color of the drainer before dissection $(\mathbf{J})$ was orange, and it then changed to green after the dissection $(\mathbf{L})$, which demonstrated that there was a considerable slowing of the nidus flow. The diagram also showed that the ROI (squares in M and N) slowly increased and then decreased (N) as compared to that observed in the predissection diagram (M). There was prolongation of the delay time, time to peak, and rise time of the ROI, and there was slowing of the speed after dissection of the nidus $(\mathrm{N})$ as compared to that observed before the dissection (M). Time(s) = time in seconds.

raphy revealed that the nidus, but not the feeders, appeared at 1 second, while the drainers appeared at 4 seconds and then disappeared at 10 seconds (Fig. 4B-D). Just prior to the removal of the nidus (Fig. 4E), ICG videoangiography showed that the nidus was still filled with ICG (Fig. 4F). The nidus was removed after ICG videoangiography confirmed there was no filling of the nidus and its drainer (Fig. 4G and $\mathrm{H}$ ).

The captured video was analyzed by Flow 800 . Prior to the dissection (Fig. 4I), the color of the drainer was orange (Fig. 4J), but it then changed to green after the dissection (Fig. 4K and L). This demonstrated a considerable slowing down of the nidus flow. As seen in a diagram for the region of interest (ROI) (Fig. 4M and N), there was a slower increase and decrease as compared to that for the predissection diagram. In addition, as compared to the predissec- tion diagram (Fig. 4M), the delay time, time to peak, and rise time of the ROI were prolonged, and the speed slowed down after dissection of the nidus (Fig. 4N).

\section{Case 3}

A 43-year-old woman presented with generalized seizures. DSA revealed a Spetzler-Martin grade II AVM in the right parietal lobe, which was fed by the middle cerebral artery and posterior cerebral artery and drained by the ascending cortical veins and vein of Labbé. The patient underwent parietotemporooccipital craniotomy, after which the nidus was removed following the feeder embolization (Fig. 5A). Intraoperative ICG videoangiography demonstrated that the feeders and nidus appeared at 1 second, while the drainers appeared at 4 seconds via the common carotid artery (CCA) (Fig. 5B and C) and the VA (Fig. 5D 

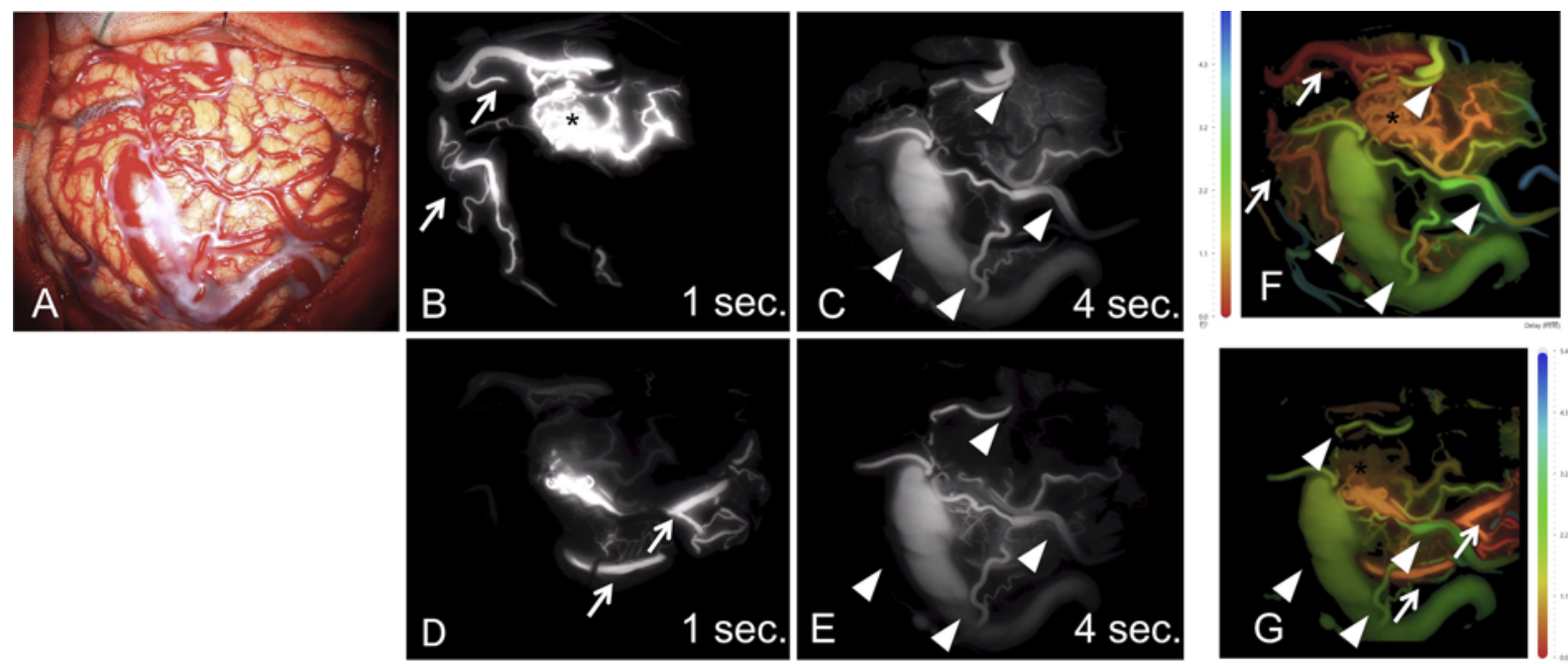

Delay
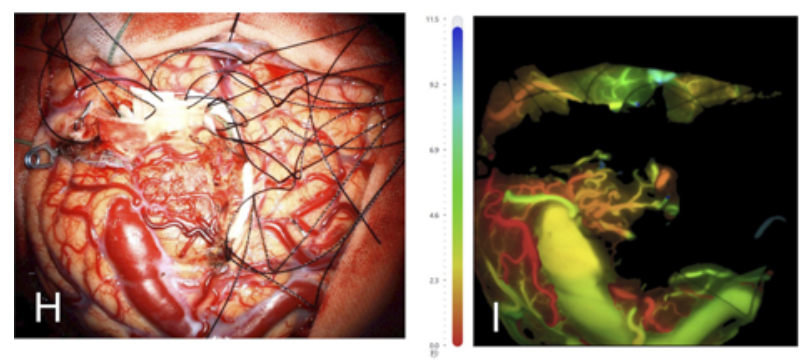

Delay

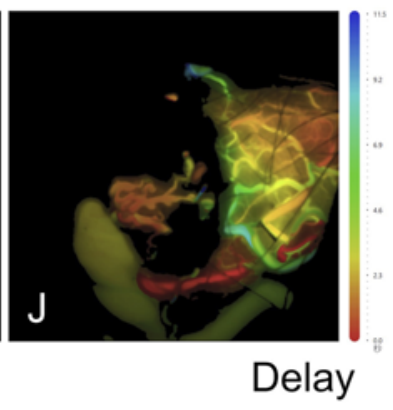

FIG. 5. Case 3. Surgical views before dissection $(\mathbf{A})$ and after partial dissection $(\mathbf{H})$ of the nidus, as well as ICG videoangiography studies obtained via the CCA (B and C) and the VA ( $D$ and $E$ ) at the time of the operation. Intraoperative ICG videoangiography revealed that the feeders and nidus appeared at 1 second, while the drainers appeared at 4 seconds $(B-E$ : arrows indicate the feeders; asterisk indicates the nidus; and arrowheads indicate the drainers). ICG videoangiography studies obtained via the CCA (F) and the VA (G) were analyzed by Flow 800 , which clearly showed the feeders, nidus, and drainers (arrows indicate the feeders; asterisk indicates the nidus; and arrowheads indicate the drainers). ICG videoangiography studies after dissection of the ventrooccipital side of the nidus obtained via the CCA $(\mathrm{I})$ and the VA $(\mathrm{J})$ were analyzed by Flow 800 , which demonstrated that the color of the main drainer changed from green to yellow, indicating there was a gradual reduction of the nidus flow.

and E). Flow 800 was used to analyze the captured movie of the ICG videoangiography performed via the CCA (Fig. 5F) and VA (Fig. 5G). This analysis clearly showed the feeders, nidus, and drainers. After dissection of the ventrooccipital side of the nidus (Fig. 5H), Flow 800 was used to analyze the captured video of the ICG videoangiography performed via the CCA (Fig. 5I) and VA (Fig. 5J). Analysis showed that the color of the main drainer changed from green to yellow, which indicated there was a gradual reduced flow in the nidus. The diagram constructed from the procedure showed that the ROIs exhibited a slow increase and decrease as compared to that observed in the predissection diagram (Fig. 6). As compared to the predissection data, the delay time, time to peak, and rise time of the ROIs were prolonged and the speed slowed down after the partial dissection of the nidus (Fig. 6).

\section{Discussion}

This study demonstrated that intraarterial ICG vid- eoangiography in AVM surgery was useful for identifying the superficial feeders, nidus, and drainers due to the brighter and higher phase contrast, as compared to that for conventional intravenous ICG videoangiography. Flow 800 analysis of color-encoded blood flow images obtained immediately after intraarterial injection of ICG made the procedure much easier to perform. In addition, the present study also demonstrated that the ICG washout time when using an intraarterial injection was within 1 minute, while this usually takes approximately 15 minutes after an intravenous injection. ${ }^{3}$ Moreover, the feeders, nidus, and drainers appeared within 10 seconds when using the intraarterial ICG injection, while it takes approximately 35 seconds when using an intravenous injection. ${ }^{3}$ Furthermore, this superiority in washout time made it possible to perform repeated videoangiography studies within a short period in order to confirm the reduced or complete lack of filling of the nidus. This allowed for a quick comparison of the two videoangiography studies due to the short span, thereby making it possible to easily recognize changes in 
A Relative intensity (\%)

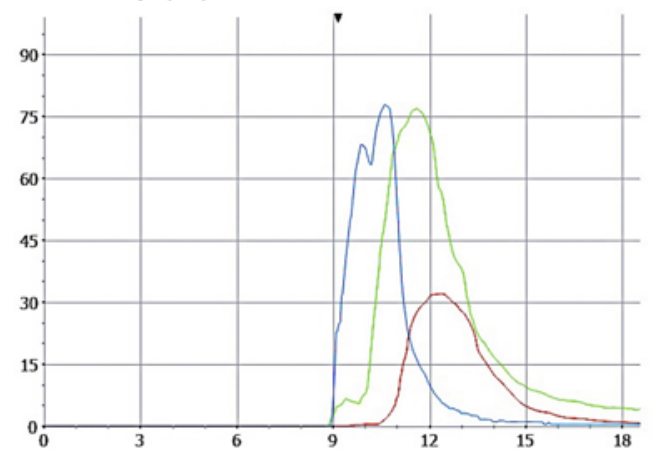

Time (sec)

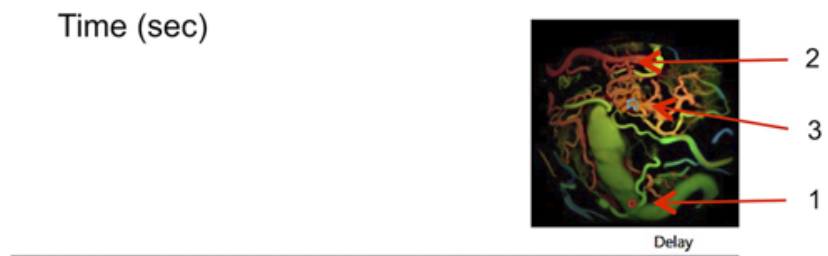

\begin{tabular}{|c|c|c|c|}
\hline ROls & 1 & 2 & 3 \\
\hline Delay time (sec) & 2.38 & 1.58 & 0.58 \\
\hline Speed (/sec) & 277 & 520 & 483 \\
\hline Time to Peak (sec) & 3.46 & 0.59 & 0.59 \\
\hline Rise Time (sec) & 0.95 & 1.15 & 1.21 \\
\hline
\end{tabular}

B Relative intensity (\%)

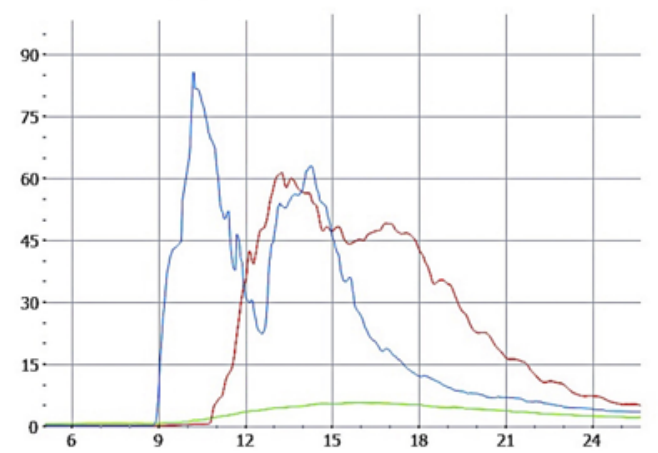

Time (sec)
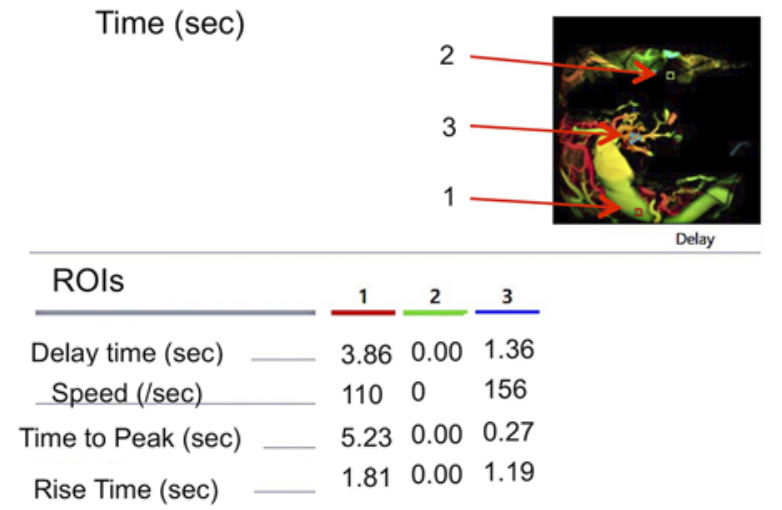

FIG. 6. Case 3. ICG videoangiography studies obtained before (A) and after (B) dissection of the ventrooccipital side of the nidus obtained via the CCA were analyzed by Flow 800 . The diagram showed that the ROls (squares in A and B) slowly increased and then decreased $(B)$ as compared to the predissection diagram $(A)$. There was prolongation of the delay time, time to peak, and rise time of ROls and the speed was slowed down (B), as compared to predissection data (A). Numbers with arrows in the ICG videoangiography studies correspond to the numbered ROIs in the tables below them.

the flow dynamics in the nidus. Flow 800 also can provide objective data such as the delay time, speed, time to peak, and rise time, which helped to confirm the reduced flow in the nidus. As compared to intravenous ICG videoangiography, intraarterial ICG videoangiography is not a time-consuming procedure, as it is possible to additionally perform intraoperative DSA in the hybrid operating room.

ICG videoangiography has been reported to be useful in bypass, aneurysm, and AVM surgeries. ${ }^{1-7}$ These previous studies have reported that ICG videoangiography has improved the simplicity, speed, and spatial resolution of the surgical view as compared to the intraoperative DSA procedure. In addition, setup for DSA requires removing the microscope in order to place the C-arm near the surgical field, while ICG videoangiography does not require any of the time-consuming procedures. In addition, since the ICG videoangiography view is the same as that seen during surgery when viewed under a microscope, this makes it easy to understand the anatomy when compared to the DSA image. Recent reports have also described the advantages of using an intraarterial injection of ICG as compared to an intravenous injection for aneurysm and spinal AVF surgery. ${ }^{8,9}$ These studies demonstrated that intraarterial injection of ICG resulted in better contrast views and a shorter ICG clearance time as compared to an intravenous injection.
Based on these advantages of intraarterial ICG injections, it appears that this procedure would be applicable for use in procedures for more complex diseases such as cerebral AVMs. Brighter and higher contrast images also can help differentiate the feeders, nidus, and drainers and, thus, separate abnormal from normal vessels. The rapid clearance of ICG additionally makes it possible to perform repeated videoangiography studies in order to review the changes in the flow dynamics of the nidus. When using an intravenous injection, ICG remains in the vessels for approximately 15 minutes, while it takes only 1 minute to clear when using an intraarterial injection. Therefore, when using intraarterial injections instead of intravenous injections, videoangiography is more similar to DSA with regard to the flow dynamics. Kono et al. reported on the first case in which an intraarterial injection of ICG was used for cerebral AVM surgery and described its usefulness. ${ }^{10}$ However, this case report did not include any Flow 800 data. In our current study, we examined the usefulness of intraarterial ICG injection during cerebral AVM surgery in various situations, which included before, during, and at end of nidus dissection, in addition to the novel use of Flow 800 data for AVM surgery in a series of cases. Our results showed that color-encoded blood flow visualization of Flow 800 was helpful in the identification of the feeder, nidus, and drainer and in the recognition of flow changes. 
Furthermore, the objective data that are also available when using Flow 800 complement these findings.

The disadvantages of both intravenous and intraarterial injection of ICG videoangiography are that they cannot detect the feeders or nidi that are covered by brain tissue. All of the feeders, nidi, and drainers, especially in cases of a residual deep nidus, can be visualized by intraoperative DSA but not by ICG videoangiography. Therefore, unlike bypass or aneurysm surgery, in our institution, routine intraoperative DSA is indispensable during AVM surgery in order to compensate for the ICG videoangiography. However, DSA images are inferior to ICG images with regard to the simplicity, speed, and spatial resolution of the surgical view, as ICG videoangiography can detect feeders on the same plane as the operative microscopic view. Thus, the operator can easily and rapidly detect feeders around the nidus without disturbing the surgical procedure. Therefore, we have now added the step of injecting ICG to the contrast medium that is administered through the catheter. Intraoperative DSA and intraarterial ICG videoangiography compensate each other, which makes the AVM surgery much safer. To avoid any thromboembolic complications, connection of all the catheters to a pressurized heparinized saline bag for the purpose of flushing is mandatory, with catheter irrigation performed every 15 minutes. As a result, our institution has not encountered any adverse coagulation events.

Adverse effects associated with ICG include arrhythmia or hypotension (with a frequency of $0.05 \%$ ) and nausea or pruritus (with a frequency of $0.2 \%$ ). ${ }^{11}$ Thus, the administration of a lower dose would likely lead to lower complication rates and a shorter clearance of ICG. However, a certain concentration level is required to maintain the fine vascular imaging. The optimal dose of ICG for intraarterial injection, especially in AVM surgery, has yet to be established at the present time. For intravenous use, $2.5 \mathrm{mg} / \mathrm{ml}$ of ICG is usually injected. In intracranial aneurysm surgery, it was reported that $0.01-0.1 \mathrm{mg} / \mathrm{ml}$ of ICG was used for the intraarterial injection,, 912 while $0.025 \mathrm{mg} /$ $\mathrm{ml}$ of ICG was used for the intraarterial injection in spinal AVF surgery. ${ }^{8}$ In the present study, we used $0.05 \mathrm{mg} / \mathrm{ml}$ of ICG without any prior examination for the purpose of determining an optimal dose for cerebral AVM surgery. This dose was higher than that reported in the previous studies. Therefore, a lower dose will need to be examined in a future study.

There are some limitations in this study. First, intraarterial injection of ICG was not mentioned in the FDA approval. Nevertheless, the injection of tiny amounts of ICG as compared to that used during an intravenous injection should be acceptable. Second, there was only a small sample size, and this was not a comparison study (such as a comparison of intravenous vs intraarterial or DSA vs ICG videoangiography, etc.); therefore, accumulation of further data and cases along with a comparison study will be necessary to definitively evaluate the safety and efficacy of this technique.

\section{Conclusions}

Intraarterial ICG videoangiography is more useful than intravenous ICG videoangiography in cerebral AVM surgery. This technique is especially effective for the identification of the feeder, nidus, and drainer to assess the nidus flow dynamics without having to use the Flow 800 analysis. However, the Flow 800 does increase the ability to more easily understand the available information. Furthermore, intraarterial ICG videoangiography can also be easily performed in any institution where intraoperative DSA is routinely performed for AVM surgeries.

\section{References}

1. Colby GP, Coon AL, Sciubba DM, et al. Intraoperative indocyanine green angiography for obliteration of a spinal dural arteriovenous fistula. J Neurosurg Spine. 2009;11(6):705-709.

2. Faber F, Thon N, Fesl G, et al. Enhanced analysis of intracerebral arterioveneous malformations by the intraoperative use of analytical indocyanine green videoangiography: technical note. Acta Neurochir (Wien). 2011;153(11):2181-2187.

3. Ng YP, King NK, Wan KR, et al. Uses and limitations of indocyanine green videoangiography for flow analysis in arteriovenous malformation surgery. J Clin Neurosci. 2013;20(2):224-232.

4. Raabe A, Nakaji P, Beck J, et al. Prospective evaluation of surgical microscope-integrated intraoperative near-infrared indocyanine green videoangiography during aneurysm surgery. J Neurosurg. 2005;103(6):982-989.

5. Suzuki K, Kodama N, Sasaki T, et al. Confirmation of blood flow in perforating arteries using fluorescein cerebral angiography during aneurysm surgery. J Neurosurg. 2007;107(1):68-73.

6. Takagi Y, Sawamura K, Hashimoto N, Miyamoto S. Evaluation of serial intraoperative surgical microscope-integrated intraoperative near-infrared indocyanine green videoangiography in patients with cerebral arteriovenous malformations. Neurosurgery. 2012;70(1)(Suppl Operative):34-43.

7. Woitzik J, Horn P, Vajkoczy P, Schmiedek P. Intraoperative control of extracranial-intracranial bypass patency by nearinfrared indocyanine green videoangiography. J Neurosurg. 2005;102(4):692-698.

8. Horie N, So G, Debata A, et al. Intra-arterial indocyanine green angiography in the management of spinal arteriovenous fistulae: technical case reports. Spine (Phila Pa 1976). 2012;37(4):E264-E267.

9. Yoshioka H, Kinouchi H, Nishiyama Y, et al. Advantage of microscope integrated for both indocyanine green and fluorescein videoangiography on aneurysmal surgery: case report. Neurol Med Chir (Tokyo). 2014;54(3):192-195.

10. Kono K, Uka A, Mori M, et al. Intra-arterial injection of indocyanine green in cerebral arteriovenous malformation surgery. Turk Neurosurg. 2013;23(5):676-679.

11. Hope-Ross M, Yannuzzi LA, Gragoudas ES, et al. Adverse reactions due to indocyanine green. Ophthalmology. 1994;101(3):529-533.

12. Gruber A, Dorfer C, Bavinzski G, et al. Superselective indocyanine green angiography for selective revascularization in the management of peripheral cerebral aneurysms. AJNR Am J Neuroradiol. 2012;33(3):E36-E37.

\section{Disclosures}

The authors report no conflict of interest concerning the materials or methods used in this study or the findings specified in this paper. 


\section{Author Contributions}

Conception and design: Shimada. Acquisition of data: all authors. Analysis and interpretation of data: Shimada. Drafting the article: Shimada. Critically revising the article: Shimada, Takagi. Reviewed submitted version of manuscript: Shimada. Approved the final version of the manuscript on behalf of all authors: Shimada.

\section{Supplemental Information}

Online-Only Content

Supplemental material is available with the online version of the article.
Supplemental Table 1. https://thejns.org/doi/suppl/10.3171/ 2020.3.JNS20319.

\section{Correspondence}

Kenji Shimada: Tokushima University Hospital, Tokushima, Japan.s_kenji1032@yahoo.co.jp. 